\title{
EFEITO DO COMPOSTO DE RESÍDUO SÓLIDO URBANO NO TEOR DE METAIS PESADOS EM SOLO E GOIABEIRA ${ }^{(1)}$
}

\author{
LEONARDO SANTOS COLLIER ${ }^{(2)}$; NELSON MOURA BRASIL DO AMARAL SOBRINHO ${ }^{(3)}$; \\ NELSON MAZUR ${ }^{(3)}$; ARY CARLOS XAVIER VELLOSO ${ }^{(4)}$
}

\begin{abstract}
RESUMO
O trabalho foi realizado no município de Cachoeiras de Macacu (RJ) com o objetivo de avaliar as várias formas dos metais $\mathrm{Cu}, \mathrm{Cr}, \mathrm{Mn}, \mathrm{Ni}, \mathrm{Pb}$ e $\mathrm{Zn}$ em solo Gleissolo pouco húmico tratado com composto de resíduos urbanos, e as concentrações desses metais nas folhas e nos frutos de goiabeira (Psidium guajava L.). Foram utilizadas três áreas com históricos distintos: área que recebeu doses de 40 t.ha $^{-1}$ de composto de resíduo sólido urbano (CRSU) na superfície por onze anos consecutivos; área com uma única aplicação de CRSU na cova; e área-controle que não utilizou CRSU e sem goiabeiras. As amostras de solo foram tomadas dentro de cada área, nas camadas de 0-5; 5-15 e 15-30 cm. A extração seqüencial dos metais contidos nas amostras $(0-5 \mathrm{~cm})$ de terra demonstrou uma tendência de redução das formas móveis ou mais biodisponíveis de metais pesados no solo com uso do CRSU. Houve aumento nos teores de metais pesados em folhas e frutos de goiabeira cultivadas em áreas que receberam CRSU; porém os teores obtidos estavam dentro da faixa considerada normal. Há necessidade de monitoramento dos teores totais de metais no solo e nas plantas, devido ao incremento dos teores de metais pesados em solos tratados com CRSU.
\end{abstract}

Palavras-chave: metais pesados, composto de resíduos sólidos urbanos, goiaba (Psidium guajava L.).

\author{
ABSTRACT \\ EFFECT OF THE URBAN SOLID WASTE COMPOST IN THE HEAVY \\ CONTENT OF METALS IN SOIL AND GUAVA ORCHARD (PSIDIUM GUAJAVA L.)
}

The work was performed in the municipal district of Cachoeiras de Macacu, Rio de Janeiro State and had as objective to obtain information on the contents of chemical forms of $\mathrm{Cu}, \mathrm{Cr}, \mathrm{Mn}, \mathrm{Ni}, \mathrm{Pb}$ and $\mathrm{Zn}$ in soil Gleissolo pouco humico treated with composted urban residues, and the concentrations in the leaves and fruits of guava (Psidium guajava L.). Three areas with different land use history were used: area that received doses of 40 t.ha $^{-1}$ of composted of urban solid residue (CRSU) in the surface for eleven consecutive years; area with only one application of CRSU in the hole; and control area, with no guava and no CRSU. The soil samples were taken from each area, in the layers of 0-5, 5-15 and $15-30 \mathrm{~cm}$. The sequential extraction of metals from the earth samples demonstrated a widespread tendency of reduction in the mobile or more bioavailable forms of heavy metals in the soil with the use of CRSU. There was

( $\left.{ }^{1}\right)$ Recebido para publicação em 12 de março e 2003 aceito em 29 de julho de 2004.

( $\left.{ }^{2}\right)$ Fundação Universitário do Tocantins (FUNITINS), Caixa Postal 66, 77410-000 Gurupi (TO). E-mail: lcollier @bol.com.br

( $\left.{ }^{3}\right)$ Departamento de Solos, Universidade Federal Rural do Rio de Janeiro (UFRRJ), 23890-000 Seropédica (RJ). E-mail: nelmoura@ufrrj.br; nelmazur@ufrrj.br

$\left({ }^{4}\right)$ Centro de Ciências e Tecnologias Agropecuárias, Universidade Estadual do Norte Fluminense (UENF), 28015-620 Campos dos Goytacazes (RJ). E-mail: velloso@uenf.br 
increase in the heavy metals contents in the leaves and fruits of guava cultivated in areas that received CRSU. However, these contents were within the normal range for economic crops. There is a need for monitoring total metal contents, in soils and plants, due to their increment in soils treated with CRSU.

Key words: heavy metals, municipal solid waste compost, guava (Psidium guajava L.).

\section{Introdução}

A compostagem do lixo urbano é empregada nas grandes e médias cidades como uma das alternativas para reduzir os problemas sociais e de contaminação do ambiente causados pela deposição desse material em vazadouros e lixões a céu aberto (Benvenuto, 1996).

A intensa utilização de adubação orgânica em áreas produtoras de hortaliças e frutas, dos chamados cinturões verdes, torna o composto de resíduo sólido urbano (CRSU) uma importante fonte de matéria orgânica, com custos acessíveis, principalmente, para os agricultores localizados próximos às usinas de compostagem.

Apesar de seus efeitos benéficos como fonte de matéria orgânica, atuando tanto nas propriedades físicas quanto químicas do solo (Bevacqua e Melano, 1994), contribuindo para o incremento de produtividade das espécies cultivadas, o uso do CRSU, todavia, é limitado pelo seu alto teor de impurezas físicas e a presença de metais pesados (MAZUR, 1997).

O conhecimento das formas químicas dos metais do solo permite melhor avaliação sobre a biodisponibilidade e mobilidade desses metais e seus riscos ao meio ambiente. CANET et al. (1997) verificaram aumento da fração trocável somente para Cd em solos calcários tratados durante sete anos com CRSU. MAZUR (1997) observou redução da fração trocável e aumento das frações extraídas com hidroxilamina (óxidos de Fe e óxido de Mn), e também que ao longo do tempo de incubação houve menor mobilidade/biodisponibilidade de $\mathrm{Ni}, \mathrm{Pb}, \mathrm{Zn}$ e $\mathrm{Cu}$ em solos incubados com CRSU durante 24 semanas.

JeEvan Rao e Shantaran (1996) mostraram que a absorção de metais pesados pelas plantas, geralmente ocorre abaixo dos níveis que causam manifestação de sintomas de toxicidade e prejuízo na produção. Entretanto, poderá interferir na qualidade dos alimentos, conforme verificado por SOARES et al. (2002).

O objetivo desse estudo foi avaliar as formas químicas de $\mathrm{Cu}, \mathrm{Zn}, \mathrm{Cr}, \mathrm{Pb}, \mathrm{Ni}$ e Mn no solo e sua acumulação em plantas de goiabeira (Psidium guajava L.) cultivadas em Gleissolo pouco húmico, que recebeu CRSU em quantidades e épocas diferentes.

\section{Material e Métodos}

O experimento foi instalado na propriedade da colônia japonesa do Funchal, localizada no município de Cachoeiras de Macacu (RJ), cujo solo pertence à classe Gleissolo pouco húmico. Nessa propriedade, foram selecionadas três glebas com histórico distintos de uso de CRSU: 1) solo cultivado com goiabeira (Psidium guajava L.) cv. Ogawa, com 12 anos de idade, adubada com CRSU durante 11 anos, em superfície, aplicado na projeção da copa $60 \mathrm{~kg}$ por planta/ano (equivalente a 40 t.ha $^{-1}$ ano $^{-1}$ ) [CRSU 11a]; 2) solo cultivado com goiabeira, com 4 anos de idade, que recebeu o CRSU em uma aplicação na cova de plantio, $60 \mathrm{~kg}$ por planta $/ \mathrm{ano}^{-1}$ [CRSU 1a]; e 3) uma áreacontrole constituída de capoeira na mesma toposseqüência em que não houve exploração agrícola intensiva (controle).

Em março de 1997, foram selecionadas quatro áreas em cada uma das três glebas anteriormente mencionadas, totalizando 12 unidades experimentais.

Em cada uma das unidades experimentais foram amostrados solos de 18 pontos delimitados por 18 goiabeiras espaçadas de 6,5 x 6,5 m. As amostras compostas de terra foram tomadas na projeção da copa da goiabeira visando coincidir com a área de aplicação do CRSU. Em cada um dos pontos foram retiradas amostras de solo na profundidade de $0-5 \mathrm{~cm} ; 5-15$ e $15-30 \mathrm{~cm}$. O teor total de $\mathrm{Ni}, \mathrm{Cr}, \mathrm{Pb}, \mathrm{Cu}, \mathrm{Zn}$ e $\mathrm{Mn}$ foi determinado em 1,0000 g de amostras de solo passadas em gral através de digestão com água-régia (mistura de três partes de ácido nítrico 65\% com uma parte de ácido clorídrico 30\%).

Realizou-se também a extração seqüencial de metais, nas amostras coletadas na profundidade $0-5 \mathrm{~cm}$, determinando o teor desses elementos nas frações trocável, óxidos de manganês e de ferro, orgânica e residual, através do método de Keller e VÉDY (1994). Também foram amostradas folhas, tomando-se 30 pares de folhas na porção mediana dos ramos em produção (NATALE, 1993), e frutos, ainda verdes, antes do ponto de colheita, nas mesmas áreas onde foram retiradas as amostras de solo, com exceção da área-controle.

Para determinar a concentração de metais pesados no material de folhas e frutos, após secagem em estufa a $65{ }^{\circ} \mathrm{C}$ por até 72 horas, 
amostras de $0,5000 \mathrm{~g}$ de material seco e finamente triturada foram submetidas à digestão nitro-perclórica (Tedesco et al, 1995). A determinação dos metais $\mathrm{Ni}$, $\mathrm{Cr}, \mathrm{Pb}, \mathrm{Cu}, \mathrm{Zn}$ e $\mathrm{Mn}$ nos extratos de solo e planta foi realizada por espectrofotometria de absorção atômica com chama ar/acetileno, em equipamento Varian AA-600.

\section{Resultados e Discussão}

Quando se comparam os teores totais dos metais pesados (Tabela 1) com os citados na literatura internacional, verifica-se na área com uso contínuo de CRSU (11 anos) que as concentrações de metais pesados encontraram-se abaixo dos níveis de referência para solos que utilizam lodo de esgoto e CRSU na Europa (Alloway, 1995) (Tabela 2). Contudo, ao se avaliar os teores de metais pesados obtidos na área-controle (Tabela 1), verificou-se o acúmulo e o potencial do CRSU em fornecer esses metais ao solo. Esse aumento nos teores de metais pesados ocorre também no solo onde foi aplicado uma dose na cova, embora em menor magnitude. Verifica-se que a maior acumulação ocorre na profundidade $0-5 \mathrm{~cm}$. Vários trabalhos têm demonstrado a baixa mobilidade de metais pesados (ADRIANO, 1992 e Alloway, 1995).

$\mathrm{Na}$ análise dos resultados da extração seqüencial das amostras de solo (Tabela 3), destaca-se a área com uso contínuo de CRSU (11 anos), com elevadas porcentagens na fração residual de quase todos os metais analisados, com exceção de $\mathrm{Zn}$ e Mn, seguida da fração óxidos de $\mathrm{Mn}$ e $\mathrm{Fe}$ para $\mathrm{Pb}, \mathrm{Ni}, \mathrm{Zn}$ e Mn e orgânica em menor proporção para $\mathrm{Cu}$ e $\mathrm{Cr}$. Esses resultados são semelhantes aos obtidos por SLOAN ET AL. (1997) em solos tratados com lodo durante 14 anos. O percentual de $\mathrm{Cu}$ ligado à fração orgânica se aproxima dos $28 \%$ obtidos por Levésque e MATHUR (1986) em solos orgânicos do Canadá.

Em função da textura arenosa e dos teores médios a elevados de matéria orgânica do solo associados à utilização do CRSU, anualmente em superfície, durante 11 anos, esperava-se um maior percentual de $\mathrm{Pb}, \mathrm{Zn}$, Ni e Mn na fração orgânica da camada superficial. Entretanto, verificou-se maior acúmulo dos referidos metais nas frações óxidos de Fe e Mn e residual.

Os resultados obtidos por MAzUR (1997), da extração seqüencial de um lote de CRSU utilizando-se também a metodologia de Keller e Védy (1994), revelaram que $\mathrm{Cu}, \mathrm{Fe}, \mathrm{Mn}, \mathrm{Ni}, \mathrm{Pb}$ e $\mathrm{Zn}$ apresentavam, respectivamente, as somas de frações trocável + óxidos de $\mathrm{Fe}$ e $\mathrm{Mn}$ de: $74 \%, 34 \%, 91 \%, 94 \%, 49 \%$ e $51 \%$ do total.

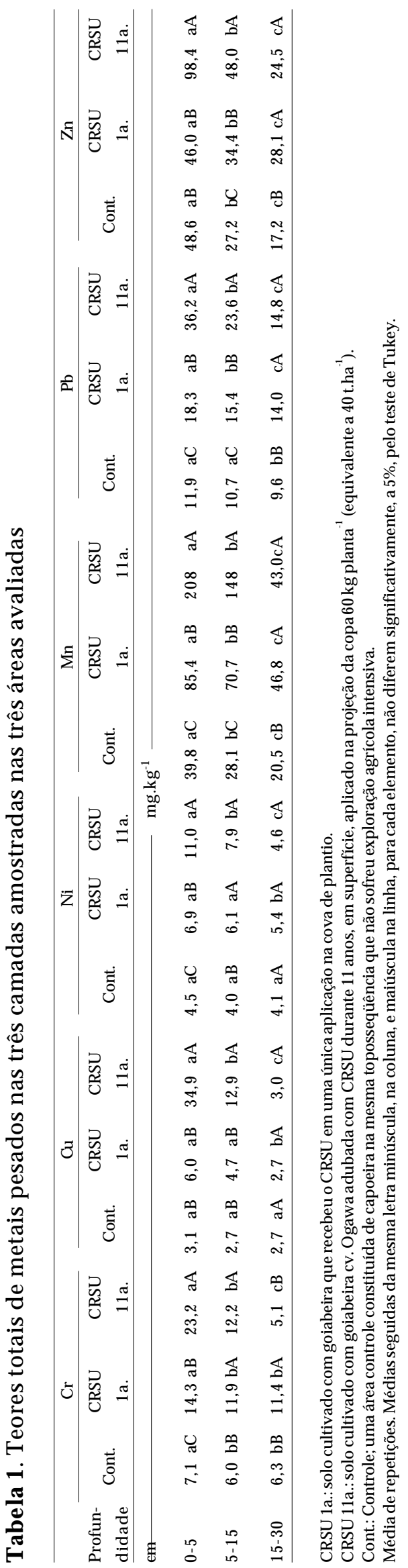

Bragantia, Campinas, v.63, n.3, p.415-420, 2004 
Tabela 2. Níveis médios anuais de metais em $\mathrm{mg} \mathrm{kg}^{-1}$, em composto de resíduo sólido urbano produzido pela COMLURB, no Rio de Janeiro-RJ, de 1983 a 1995, e níveis de metais em lodo de esgoto e solos de várias partes do mundo

\begin{tabular}{|c|c|c|c|c|c|c|c|}
\hline Elemento & $1983 / 90$ & 1992 & 1993 & 1994 & 1995 & $\begin{array}{l}\text { Concentração } \\
\text { crítica no lodo }\end{array}$ & $\begin{array}{c}\text { Níveis médios } \\
\text { no solo }\end{array}$ \\
\hline $\mathrm{Cd}$ & 5 & 2,2 & 2,8 & 2,0 & 1,5 & 5 & 0,30 \\
\hline $\mathrm{Cr}$ & 274 & 41 & 90 & 80 & 45 & 150 & 20 \\
\hline $\mathrm{Cu}$ & 469 & 295 & 199 & 425 & 196 & 100 & 20 \\
\hline $\mathrm{Hg}$ & 2,8 & 1,2 & 5,7 & 0,13 & 0,06 & 4 & - \\
\hline $\mathrm{Mn}$ & 480 & 75 & 215 & 260 & 119 & - & 850 \\
\hline $\mathrm{Ni}$ & 150 & 37 & 23 & 40 & 64 & 50 & 40 \\
\hline $\mathrm{Pb}$ & 477 & 274 & 278 & 340 & 243 & 500 & 10 \\
\hline $\mathrm{Zn}$ & 999 & 227 & 480 & 710 & 370 & 1000 & 50 \\
\hline
\end{tabular}

$\left(^{1}\right)$ Limites para concentração de metais pesados no composto de lixo urbano adotados para uso agrícola por alguns países da Europa, com base no material seco (Berton, 1996; Alloway, 1995).

$\left(^{2}\right)$ Concentração de metais em solos de áreas não industrializadas de várias partes do mundo (ADRIANO, 1992).

Tabela 3. Fracionamento de cobre, zinco, níquel, chumbo, cromo e manganês nas diferentes formas químicas, na profundidade de $0-5 \mathrm{~cm}$ (média de 4 repetições)

\begin{tabular}{|c|c|c|c|c|c|}
\hline Tratamento & Trocável & Óxidos & Orgânico & Residual & Total \\
\hline & \multicolumn{5}{|c|}{$\mathrm{mg} \cdot \mathrm{kg}^{-1}$} \\
\hline & \multicolumn{5}{|c|}{ Cobre } \\
\hline Controle $^{(1)}$ & $0,00(0,00)^{(4)} \mathrm{a}$ & $0,18(5,81) b$ & $1,13(36,45) \mathrm{c}$ & $1,79(57,74) b$ & $3,1 \mathrm{~b}$ \\
\hline CRSU $1 \mathrm{a}^{(2)}$ & $0,00(0,00) a$ & $0,63(10,50) a$ & $3,5(58,33) \mathrm{b}$ & $1,87(31,17) b$ & $6,0 \mathrm{~b}$ \\
\hline \multirow[t]{2}{*}{ CRSU $11 \mathrm{a}^{\cdot(3)}$} & $0,00(0,00) a$ & $0,66(1,89) a$ & $8,9(25,50) a$ & $25,34(72,61) a$ & $35,0 \mathrm{a}$ \\
\hline & \multicolumn{5}{|c|}{ Zinco } \\
\hline Controle & $0,24(0,49) \mathrm{a}$ & $6,12(12,59) \mathrm{c}$ & $3,06(6,30) b$ & $39,18(80,62) a$ & $49,0 \mathrm{~b}$ \\
\hline CRSU 1a. & $0,04(0,09) \mathrm{b}$ & $28,06(61,00) \mathrm{b}$ & $11,96(26,00) a$ & $5,94(12,91) \mathrm{c}$ & $46,0 \mathrm{~b}$ \\
\hline \multirow[t]{2}{*}{ CRSU 11a. } & $0,20(0,20) a$ & $71,04(72,20) a$ & $8,07(8,20) a$ & $19,09(19,40) b$ & $98,0 \mathrm{a}$ \\
\hline & \multicolumn{5}{|c|}{ Níquel } \\
\hline Controle & $0,00(0,00) \mathrm{a}$ & $0,00(0,00) b$ & $0,00(0,00) b$ & $4,50(100,00) b$ & $4,5 \mathrm{c}$ \\
\hline CRSU 1a. & $0,17(2,50) \mathrm{a}$ & $0,12(1,80) b$ & $1,13(16,40) a$ & $5,48(79,40) \mathrm{b}$ & $6,9 \mathrm{~b}$ \\
\hline \multirow[t]{2}{*}{ CRSU 11a. } & $0,00(0,00) \mathrm{a}$ & $1,46(13,30) a$ & $1,57(14,30) a$ & $7,98(72,50) a$ & $11,0 \mathrm{a}$ \\
\hline & \multicolumn{5}{|c|}{ Chumbo } \\
\hline Controle & $0,00(0,00) \mathrm{a}$ & $1,79(15,00) \mathrm{c}$ & $3,70(31,10) a$ & $6,41(53,90) b$ & $12,0 \mathrm{c}$ \\
\hline CRSU 1a. & $0,00(0,00) \mathrm{a}$ & $5,29(28,90) \mathrm{b}$ & $5,44(29,70) a$ & $7,58(41,40) b$ & $18,0 \mathrm{~b}$ \\
\hline \multirow[t]{2}{*}{ CRSU 11a. } & $0,00(0,00) \mathrm{a}$ & $9,59(26,50) a$ & $4,49(12,40) a$ & $22,12(61,10) a$ & $36,0 \mathrm{a}$ \\
\hline & \multicolumn{5}{|c|}{ Cromo } \\
\hline Controle & $0,28(3,90) a$ & $2,66(37,50) a$ & $0,53(7,50) b$ & $3,63(51,10) \mathrm{c}$ & $7,1 \mathrm{c}$ \\
\hline CRSU 1a. & $0,24(1,70) \mathrm{a}$ & $1,32(9,20) a$ & $2,47(17,30) a$ & $10,27(71,80) b$ & $14,0 \mathrm{~b}$ \\
\hline \multirow[t]{2}{*}{ CRSU 11a. } & $0,21(0,90) \mathrm{a}$ & $1,30(5,60) \mathrm{a}$ & $5,34(23,00) a$ & $16,33(70,40) a$ & $23,0 \mathrm{a}$ \\
\hline & \multicolumn{5}{|c|}{ Manganês } \\
\hline Controle & $3,90(9,80) a$ & $31,72(79,70) \mathrm{b}$ & $2,11(5,30) \mathrm{c}$ & $2,11(5,30) \mathrm{c}$ & $40,0 \mathrm{c}$ \\
\hline CRSU 1a. & $0,60(0,70) \mathrm{b}$ & $33,31(39,00) b$ & $16,23(19,00) b$ & $35,27(41,30) a$ & $85,0 \mathrm{~b}$ \\
\hline CRSU 11a. & $0,83(0,40) \mathrm{b}$ & $157,87(75,90) a$ & $24,34(11,70) a$ & $24,96(12,00) b$ & $208,0 \mathrm{a}$ \\
\hline
\end{tabular}


Comparando esses percentuais com aqueles obtidos na área com uso contínuo de CRSU, os elementos $\mathrm{Cu}$ e Ni obtiveram redução nas frações mais biodisponíveis; $\mathrm{Pb}$ e $\mathrm{Mn}$ não apresentaram alteração, e o Zn elevou o percentual de formas lábeis no solo.

A associação do Fe procedente do CRSU com a matéria orgânica na camada superficial da área com uso contínuo do composto pode ter levado à formação de óxidos de Fe e Mn de baixa solubilidade. Portanto, pouco redutíveis com hidroxilamina, ou novas superfícies adsorventes intermediadas por ligantes orgânicos (KeEFer e SiNGH, 1986) de difícil oxidação, inclusive pela água oxigenada, ocorrendo, predominantemente, na fração residual e óxidos de $\mathrm{Fe}$ e Mn. A distribuição percentual nas diferentes frações químicas de $\mathrm{Cu}, \mathrm{Ni}, \mathrm{Pb}$ e $\mathrm{Zn}$ da área que utilizou o CRSU apenas na cova, por ocasião do plantio das mudas, apresentou menor acúmulo na fração residual, causando maior biodisponibilidade em relação à área com uso contínuo de CRSU. Na áreacontrole, os elementos $\mathrm{Ni}$ e Zn, com menor intensidade, apresentaram maior fração residual que a área com uso contínuo de CRSU, confirmando as observações de CHLOPECKA et al. (1996) para o comportamento de metais em áreas de menor influência antropogênica.

Os teores de metais analisados nas folhas e frutos (Tabela 4) estiveram dentro da faixa considerada normal de teores apresentados por ADRIANo (1992) para folhas e Alloway (1995) para frutos para a maioria das culturas de interesse econômico.

Tabela 4. Concentreções de metais pesados nas folhas e frutos de goiabeira, em $\mathrm{mg} \mathrm{kg}^{-1}$, as diferentes áreas estudadas e valores de referência

\begin{tabular}{|c|c|c|c|c|c|c|}
\hline Tratamentos & $\mathrm{Ni}$ & $\mathrm{Cr}$ & $\mathrm{Pb}$ & $\mathrm{Cu}$ & $\mathrm{Zn}$ & Mn \\
\hline & \multicolumn{6}{|c|}{ mg.kg-1 } \\
\hline & \multicolumn{6}{|c|}{ FOLHA } \\
\hline CRSU 1ano & $17,70 \mathrm{a}$ & $1,60 \mathrm{a}$ & 4,88 a & $10,72 \mathrm{a}$ & $21,50 \mathrm{~b}$ & $64,30 \mathrm{~b}$ \\
\hline CRSU 11anos & $13,10 \mathrm{~b}$ & $1,32 \mathrm{~b}$ & $3,21 b$ & 8,64 b & $29,00 \mathrm{a}$ & $97,90 \mathrm{a}$ \\
\hline \multirow[t]{2}{*}{ Níveis de referência ${ }^{(1)}$} & 23 & 16 & 30 & 13 & 55 & 262 \\
\hline & \multicolumn{6}{|c|}{ FRUTO } \\
\hline CRSU 1ano & $1,13 \mathrm{a}$ & $2,85 \mathrm{a}$ & $5,10 \mathrm{~b}$ & $3,21 \mathrm{a}$ & $9,04 \mathrm{a}$ & $1,36 \mathrm{~b}$ \\
\hline CRSU 11anos & $0,75 \mathrm{a}$ & $2,06 \mathrm{a}$ & $6,90 \mathrm{a}$ & $2,57 \mathrm{~b}$ & 7,98 a & $4,10 \mathrm{a}$ \\
\hline Níveis de referência ${ }^{(2)}$ & 5 & 14 & 20 & 6 & 14 & 23 \\
\hline
\end{tabular}

(1) Níveis de referência segundo Adriano (1992)

$\left(^{2}\right)$ Níveis de referência segundo Alloway (1995)..

Médias seguidas da mesma letra minúscula, na coluna, para cada elemento, e para cada parte da planta não diferem significativamente, ao nível de 5\%, pelo teste Tukey.

\section{Conclusões}

1. Houve incremento dos teores de metais pesados na camada superficial dos solos das duas áreas que receberam aplicação de composto de resíduos sólidos urbanos, não atingindo níveis críticos citados pela literatura internacional.

2. O uso do composto de resíduos sólidos urbanos nas doses aplicadas, dentro do sistema de manejo do solo avaliado, não representou risco de acumulação de metais pesados nas folhas e frutos das plantas cultivadas acima dos padrões encontrados até então pela literatura internacional.

\section{Referências}

ADRIANO, D.C. Biogeochemistry of trace metals. Boca Raton: Lewis Publishers, 1992. 513p.

ALLOWAY, B.J. Heavy metals in soils. London: Blackie A e P, 1995. 368p.

BENVENUTO, C. Técnicas de remediação de aterros e lixões. In : CONGRESSO LATINO-AMERICANO DE CIÊNCIA DO SOLO, 13., Águas de Lindóia, 1996. Anais... Comissão de Poluição do Solo e Qualidade do Ambiente. (CD-ROM)

BERTON, R.S. Utilização do composto de lixo na agricultura. In: CONGRESSO LATINO-AMERICANO DE CIÊNCIA DO SOLO, 13., 1996, Águas de Lindóia. Anais... Comissão de Poluição do Solo e Qualidade do Ambiente, 1996. (CD-ROM) 
BEVACQUA, R.F.; MELANO, V.J. Cumulative effects of sludge compost on crop yields and soil properties. Communication in Soil Science Plant Analysis, New York, v.25, n.3-4, p.395406, 1994.

CANET, R.; POMARES, F.; TARAZONA, F. Chemical extractability and availability of heavy metals after seven years application of organic wastes to a citrus soil. Soil Use and Management, Aberdeen, v.13, p.117-121, 1997.

CHLOPECKA, A.; BACON, J.R.; WILSON, M.J.; KAY, J. Forms of cadmium, lead and zinc in contaminated soils from southwest Poland. Journal of Environmental Quality, Madison, v.25, p.769-799, 1996.

JEEVAN RAO, K.; SHANTARAM, M.V. Effect of urban solid wastes on dry matter yield, uptake of micronutrients and heavy metals by maize plants. Journal of Environmental Biology, Muzaffarnagar, v.17, n.1, p.25-32, 1996.

KEEFER, R.F.; SINGH, R.N. Correlation of metal-organic fractions with soil properties in sewage-sludge amended soils. Soil Science, Madison, v.142, n.1, p.20-26, 1986.

KELLER, C.; VÉDY, J.C. Heavy metals in the environment: distribution of cooper and cadmium fractions in two forest soils. Journal of Environmental Quality, Madison, v.23, p.987999, 1994.

LEVÉSQUE, M.; MATHUR, S.P. The influence of soil properties, iron, manganese, and zinc on the level and distribution of cooper. Soil Science, Madison, v.142, n.3, p.153$163,1986$.
MAZUR, N. Níquel, chumbo, zinco e cobre em solos que receberam composto de resíduos sólidos urbanos. 1997. 129 f. Tese (Doutorado em Solos e Nutrição de Plantas) Universidade Federal de Viçosa.

NATALE, W. Diagnose da nutrição nitrogenada e potássica em duas cultivares de goiabeira (Psidium guajava L.) durante três anos. 1993. 153 f. Tese (Doutorado em Nutrição Mineral de Plantas). Escola Superior de Agricultura Luiz de Queiroz ESALQ-USP, Piracicaba.

SLOAN, J.J., DOWDY, R.W., DOLAN, M.S., LINDEN, D.R. Longterm effects of biosolids applications on heavy metal bioavailability in agricultural soils. Journal of Environmental Quality, Madison, v.27 n.4, p.966-974, 1997.

SOARES, F.; AMARAL SOBRINHO, N.M.B; MAZUR,N. Influência de diferentes manejos agrícolas na distribuição de metais pesados no solo e em plantas de tomate. Revista Brasileira de Ciência do Solo, Viçosa, v.26, p.535-542, 2002.

TEDESCO, M.J.; GIANELLO, C.; BISSANI, C.A.; BOHNEN, H.; VOLKWEISS, S.J. Análise de solo, plantas e outros materiais. 2.ed. Porto Alegre: Departamento de Solos, UFRGS, 1995. 174p. (Boletim técnico n.5). 